\title{
Miscibility Gap in the Microbial Fitness Landscape
}

\author{
F. N. Braun, ${ }^{1}$ S. Paulsen, ${ }^{1}$ R. P. Sear, ${ }^{2}$ and P. B. Warren ${ }^{3}$ \\ ${ }^{1}$ Institute of Medical Biology, University of Tromso, N-9037 Tromso, Norway \\ ${ }^{2}$ Department of Physics, University of Surrey, Guildford, Surrey GU2 7XH, United Kingdom \\ ${ }^{3}$ Unilever R\&D Port Sunlight, Bebington, Wirral CH63 3JW, United Kingdom
}

(Received 7 December 2004; published 5 May 2005)

\begin{abstract}
It is shown from molecular statistical considerations that a demixing instability exists in the moment space of a microbial protein expression profile. Although avoidance of demixing is generally requisite for biological function, a comparison with proteomic and genomic data suggests that many microbes lie close to the onset of this instability. Over evolutionary time scales, straying too close or into the immiscible domain may be associated with intracellular compartmentalization.
\end{abstract}

DOI: 10.1103/PhysRevLett.94.178105

PACS numbers: 87.16.-b

Molecular statistical approaches to demixing thermodynamics have long focused on industrially important contexts such as polymer blends, colloids and crude oil [1]. Similar avenues might also present useful insights into the intracellular thermodynamics of microbial organisms. Odijk [2] for example, proposes an equilibrium thermodynamic view of the bacterial nucleoid according to which, under conditions of excess salt, DNA tends to reversibly collapse and demix from the cytosol proteome.

The proteome itself features only as a secondary focus in Odijk's particular analysis, but it is also of interest to examine how from a statistical mechanical perspective microbes apparently manage to avoid a similar intraproteomic demixing effect [3]. Expressed proteins of course do not disperse in perfectly miscible souplike fashion, but we can reasonably suppose that they must remain essentially miscible in respect of their macroscopic phase behavior. It is known for molecular mixtures in general that miscibility is sensitive to low moments of the size distribution [4], so we might anticipate that microbial intracellular stability depends analogously on moments of the proteomic expression level profile with respect to sequence length. Our principal objective here is to demonstrate this more explicitly within a model framework.

Consider a crude molecular statistical formulation of the Helmholtz free energy $F=U-T S$ describing the expressed protein ensemble, where $U$ and $S$ denote, respectively, internal energy and entropy at temperature $T$. We assume a continuous distribution $\phi(l)$ over length $l$ in amino acid residues. With $\rho$ the total protein number density, $\rho \phi(l) d l$ is the concentration in the cytosol having length between $l$ and $l+d l$. Assuming proteins with the same $l$ can be considered indistinguishable with respect to their mutual interactions, we can then write for the entropy density over volume $V$ of the cytosol

$$
S / V=-k_{B} \rho \int \phi(l)\{\ln [\rho \phi(l)]-1\} d l
$$

where $k_{B}$ is Boltzmann's constant.
Next we assume that the dominant contribution to the internal energy $U$ comes from nonspecific adhesive interaction between proteins. For a system of monodisperse adhesive well particles $U / V \simeq-2 \pi \epsilon \rho^{2} a d^{2}$, where $d$ is the particle diameter, $a$ is the well width, and $\epsilon$ is its depth. In this spirit, we write

$$
U / V=-2 \pi \epsilon \rho^{2} a^{3}\left\langle l^{2 / 3}\right\rangle,
$$

where $\langle\ldots\rangle$ denotes the distribution-averaged moment. Here we have identified $a$ with the amino acid length scale, and set $d=a l^{1 / 3}$ to represent a compact protein comprising $l$ residues.

To look for a miscibility gap in the parameter space of this description, we construct the matrix of second partial derivatives of $F$ with respect to dimensionless moment densities $\nu_{0}=\rho a^{3}$ and $\nu_{1}=\rho a^{3}\left\langle l^{2 / 3}\right\rangle$. A spinodal locus of demixing will occur when the lowest eigenvalue of this matrix goes to zero, the corresponding eigenvector giving the unstable spinodal fluctuation mode which begins to grow unchecked as the locus is crossed.

Adopting the Legendre transform method outlined in [5] to treat the entropic part, we obtain

$$
\begin{aligned}
\nu_{0} \partial^{2} \tilde{F} / \partial \nu_{0}^{2}= & \left\langle l^{4 / 3}\right\rangle\left[\left\langle l^{4 / 3}\right\rangle-\left\langle l^{2 / 3}\right\rangle^{2}\right]^{-1}, \\
\nu_{0} \partial^{2} \tilde{F} / \partial \nu_{1}^{2}= & {\left[\left\langle l^{4 / 3}\right\rangle-\left\langle l^{2 / 3}\right\rangle^{2}\right]^{-1} } \\
\nu_{0} \partial^{2} \tilde{F} / \partial \nu_{0} \nu_{1}= & -\left\langle l^{2 / 3}\right\rangle\left[\left\langle l^{4 / 3}\right\rangle-\left\langle l^{2 / 3}\right\rangle^{2}\right]^{-1} \\
& -2 \pi \epsilon \rho a^{3} / k T,
\end{aligned}
$$

where $\tilde{F}=a^{3} F /(V k T)$ is a convenient dimensionless scaling of the free energy. Hence from the zero determinant condition $\partial^{2} \tilde{F} / \partial \nu_{0}^{2} \partial^{2} \tilde{F} / \partial \nu_{1}^{2}=\left(\partial^{2} \tilde{F} / \partial \nu_{0} \partial \nu_{1}\right)^{2}$, we have for the spinodal

$$
\left(\pi \epsilon \rho a^{3} / k T\right)\left\langle l^{2 / 3}\right\rangle+\left(\pi \epsilon \rho a^{3} / k T\right)^{2}\left[\left\langle l^{4 / 3}\right\rangle-\left\langle l^{2 / 3}\right\rangle^{2}\right]=1 / 4 .
$$

Since the total protein volume fraction $\eta \simeq 15 \%$ is a fairly universal feature of a microbial cytosol, it is useful to recast this expression making use of the approximate rela- 
tion $\eta \simeq \rho a^{3}\langle l\rangle$

$$
(\pi \eta \epsilon / k T)\langle l\rangle\left\langle l^{2 / 3}\right\rangle+(\pi \eta \epsilon / k T)^{2}\left[\left\langle l^{4 / 3}\right\rangle-\left\langle l^{2 / 3}\right\rangle^{2}\right]=\langle l\rangle^{2} / 4 .
$$

In Fig. 1 we have plotted this constant volume fraction result over the moment space of a log-normal distribution. A miscibility gap is reached with decreasing $\langle l\rangle$, depending only very weakly on the reduced standard deviation $\delta=$ $\sigma /\langle l\rangle$, and tending to increase in size with increasing adhesive strength $\epsilon / k T$. The calculation sets a physically plausible $\epsilon=3.5 \mathrm{kT}$, roughly reflecting literature estimates of the free energy gain of burying a surface-exposed hydrophobic residue in a protein-protein contact [6].

The predicted low sensitivity to polydispersity is noteworthy. It indicates that, in respect to phase separation, the cytosol is well represented by a hypothetical monodisperse distribution comprising proteins of a single length, a minimum length being required for stability [7]. The miscibility gap can be regarded simultaneously as a precipitous gap in the fitness landscape governing microbial phenotype. Hence the miscibility criterion on length generates an evolutionary pressure in favor of larger proteins. It is interesting to note that, in as much as (via more general treatment of the protein-protein interaction parameter $\epsilon$ ) the miscibility gap will tend to shift with temperature, salinity, and $p \mathrm{H}$, this nature of associated evolutionary pressure is coupled to the environment.

In living microbial organisms, proteins are constantly synthesized, degraded, resynthesized. This turnover gen-

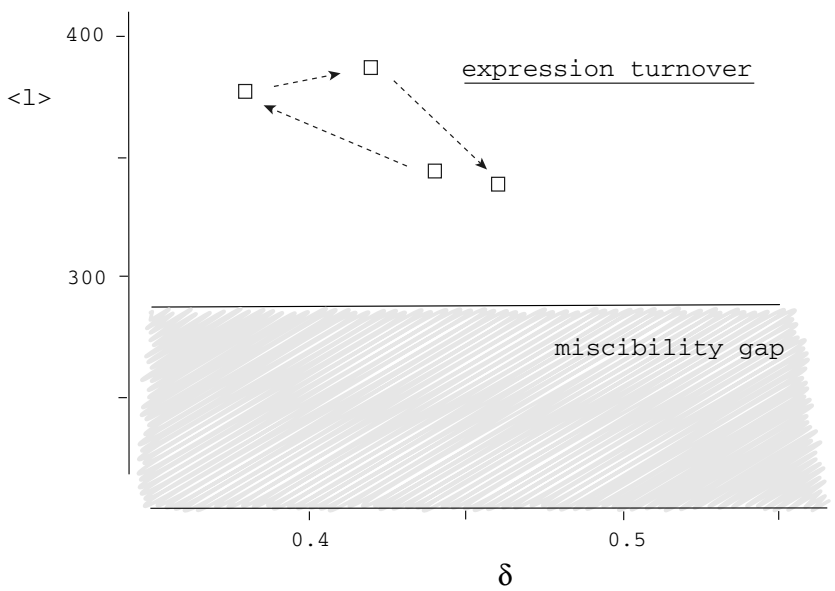

FIG. 1. Model prediction of the miscibility gap (shaded) in the space of low moments of the protein expression profile $\phi(l)$, i.e., mean sequence length $\langle l\rangle$ vs reduced standard deviation $\delta=$ $\sigma /\langle l\rangle$. For experimental comparison, the points present samples of the protein expression profile of Vibrio salmonicida under four different growth conditions. The samples monitor the relative abundance of 24 protein species randomly chosen from a 2D gel electrophoretic map [8]. During the lifespan of an organism, points such as these would typically fall along some complex dynamical trajectory (arrows, figurative only) of environmental response and stochastic fluctuation. erates a temporal dynamics over the moment space of our model, which can be explored experimentally by proteomic techniques such as $2 \mathrm{D}$ gel electrophoresis. By way of illustration, we have included in Fig. 1 some results from proteomic analysis of the bacterium Vibrio salmonicida responding to different growth conditions [8]. Although clearly there is scope for further and more systematic investigation along such lines, these results are broadly consistent with the scenario we are suggesting, in so far as turnover remains confined to the predicted miscible domain.

From a genome-evolutionary point of view, an intriguing possibility is that the fairly localized nature of the turnover in moment space which is in evidence here might loosely correlate with the corresponding distribution of open reading frames (ORFs) along the genome. That is, the number of proteins of a given length expressed in the cytosol at any given time is weakly proportional to the number of proteins of that length in the organism's genome. For example, the mean position of the points in Fig. 1 is $\langle l\rangle=362, \delta=$ 0.43 , as compared with $\langle l\rangle=316, \delta=0.68$ for the $V$. salmonicida ORF length distribution. This idea is pursued in Fig. 2 where we have plotted the moment space coordinates of several of the microbial genomes available from public databases. The dataset clusters in a marginally stable band along the gap's edge, the width of the band reflecting a plausible margin of selective error $\delta \epsilon \sim k T / 2$.

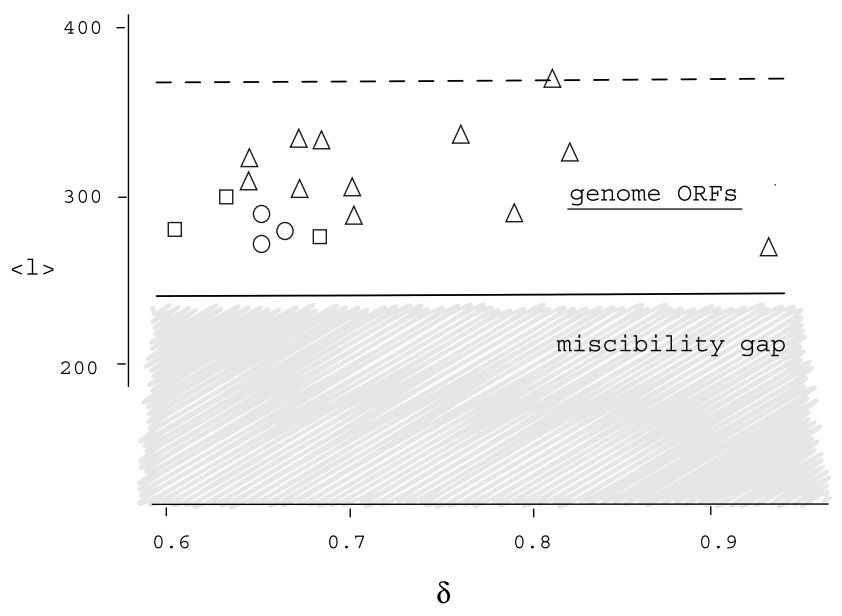

FIG. 2. Whole-genome ORF length distributions appear to cluster in a "marginal band" along the edge of the miscibility gap. The lower and upper bounds shown here are the spinodal limits calculated at $\epsilon=3.3 \mathrm{kT}$ and $3.8 \mathrm{kT}$ respectively. Bacterial mesophiles (triangles, left to right) $H$. influenzae, E. coli, B. burgdorferi, S. pyogenes, R. prowaszekii, L. lactis, L. innocua, M. leprae, N. meningitidis, M. pulmonis, Nostoc sp., and $X$. fastidiosa; Archaeal thermophiles (squares) S. solfataricus, T. acidophilum, and M. thermoautotrophicum; Archaeal hyperthermophiles (circles) P. furiosus, M. kandleri, and A. pernix. All the genomes of this dataset are log-normal, according to the criterion that excess kurtosis is significant at a chi-square test threshold $P=0.1$. 
(Here we have in mind that $k T$ limits the resolution of mutational changes in protein-protein interaction energies on which negative selection can operate.) Thus it appears feasible that, beyond directly constraining protein expression, miscibility might also contribute to shaping the genomic ORF length distribution.

Up to this point, we have considered the gap to be strictly unviable in respect of biological function, hence evolutionarily deleterious. Inside the gap, the energy/entropy balance of the cytosol is upset to the extent that it is no longer possible to express its proteome without a significant fraction spontaneously condensing out into new thermodynamically distinct compartments, which ordinarily we expect to impede function. The presumably rather small probability that a viable compartmentalization can occur inside the gap nevertheless merits consideration, in so far as it might have some bearing on the evolutionary transition from prokaryote to eukaryote (Fig. 3). In particular, there is currently no clear consensus on the origin of the eukaryote nucleus. A symbiotic merger between prokaryote strains is a strong contender, but a number of

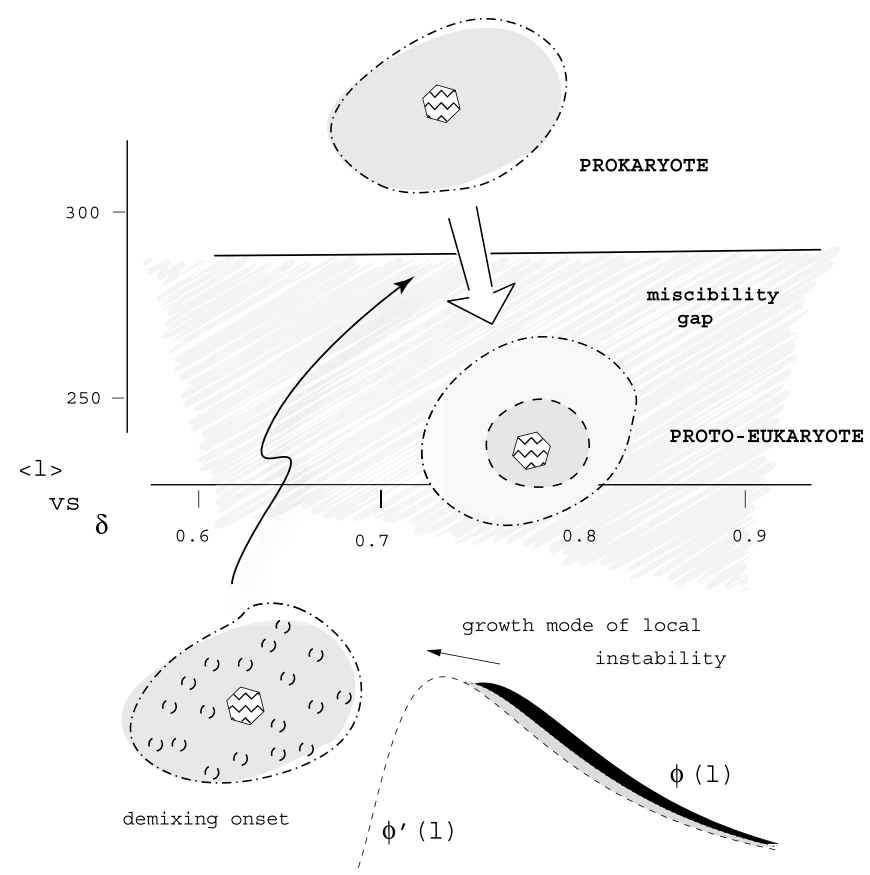

FIG. 3. Thermodynamic route to intracellular compartmentalization. If a prokaryote enters the gap, local compositional fluctuations become unstable in the cytosol, growing and condensing into at least one thermodynamically distinct nascent compartment. The unstable mode of fluctuation along the edge of the gap is shown bottom right. This points towards a nascent compartment in which the molecular weight distribution has lower $\langle l\rangle$ and slightly higher variance than the original cytosol (also lower density $\rho$, not shown). In the majority of instances, a compartment formed in this way is unlikely to be functionally viable, nor does it necessarily envelope the prokaryote nucleoid (hexagon). alternative possible origins appear similarly plausible, notably the involvement of viruses [9]. Although supported to varying extents by sequence comparative analysis, it is less clear how these competing hypotheses deal with existing examples of prokaryote strains in which the DNAcomprising nucleoid sits within a distinct membranebound intracellular compartment: in effect a crude nuclear body, but lacking further attributes of the modern eukaryote nucleus (nuclear pores, nucleosomes, etc.) [10]. If these strains reflect a first evolutionary step in nuclear development taken by ancestral proto-eukaryotes, then a thermodynamic explanation along the present lines seems a plausible alternative to the existing proposals.

In addition to a mutational mode driving the transition (i.e., changes in the expressed protein length distribution), our approach suggests a relatively straightforward selective rationale. By putting different proteins into different compartments, an organism can eliminate or at least alleviate the miscibility constraint. It thereby achieves greater freedom to evolve, asserting biochemical fitness advantages which would otherwise be inaccessible to a simple prokaryote.

Alternatively in our approach, compartmentalization can also be induced by changes in the environmental engines of microbial diversity-temperature, salinity, and $p \mathrm{H}$. As the stability threshold responds to environmental fluctuations, it is conceivable that microbial strains, finding themselves deep inside the miscibility gap, are forced to test the functional viability of spontaneously formed compartments on a fairly regular basis.

Overall, the statistical mechanical model and interpretation of data we have presented here ignore numerous caveats. For example, besides the obvious limitations of the ORF-cytosol correlation ansatz, we have not filtered off from the dataset putative membrane ORFs which are not expressed in the cytosol. A real world cytosol is never sensu stricto in the ergodic statistical mechanical equilibrium which is tacitly assumed in the miscibility analysis. Moreover, we have neglected all specific details of protein-protein interaction and molecular recognition. Nevertheless, despite this crudeness of approximation on various fronts, it seems clear that the general phenomenology of intracellular miscibility is capable of strongly influencing microbial proteomics and evolution.

[1] R. T. DeHoff, Thermodynamics in Material Science (McGraw-Hill, New York, 1992).

[2] T. Odijk, Biophys. Chem. 73, 23 (1998).

[3] R. P. Sear and J. A. Cuesta, Phys. Rev. Lett. 91, 245701 (2003).

[4] P. Sollich, J. Phys. Condens. Matter 14, R79 (2002).

[5] P. B. Warren, Europhys. Lett. 46, 295 (1999).

[6] B. Vallone et al., Proc. Natl. Acad. Sci. U.S.A. 95, 6103 (1998). For the idealized adhesive well fluid motivating our expression for $U$, this is a rather high well depth, 
sufficient to drive nonequilibrium effects. It can be argued that the "true depth", say $w$, of the equivalent adhesive well system is smaller, $w=k T \ln (1+\epsilon / k T) \simeq 1.5 k T$

[7] Note that this result is not far removed from the conventional wisdom that larger proteins, because of their smaller surface to volume ratio favoring burial of hydrophobic groups, often tend to be more soluble. See, e.g., D.S.
Goodsell and A.J. Olson, Trends Biochem. Sci. 18, 65 (1993).

[8] Experimental protocols and detailed results for V. salmonicida will be published elsewhere.

[9] E. Pennisi, Science 305, 766 (2004).

[10] M. R. Lindsay et al., Arch. Microbiol. 175, 413 (2001). 